\title{
Capillary force lithography with impermeable molds
}

\author{
Hyunsik Yoon, Tae-il Kim, Sejin Choi, ${ }^{a)}$ Kahp Y. Suh, ${ }^{\text {b) }}$ M. Joon Kim, and Hong H. Lee ${ }^{c}$ \\ School of Chemical and Biological Engineering, Seoul National University, Seoul 151-742, Korea
}

(Received 11 August 2005; accepted 26 April 2006; published online 21 June 2006)

\begin{abstract}
Capillary force lithography (CFL) with impermeable mold is presented. For the CFL to be operative over large area, either the mold or the substrate has to be flexible. With a silicon wafer mold and a flexible substrate, a repeated line and space pattern with a spacing of $30 \mathrm{~nm}$ is shown to be well patterned. With a flexible mold and a hard substrate, a similar pattern with a spacing of $60 \mathrm{~nm}$ is demonstrated by CFL. The flexibility is needed for the intimate contact that is required between the mold and the substrate for the capillarity to take hold over large area. The forte of CFL with impermeable mold lies in the fact that the driving force for the patterning, which is capillary force, increases with decreasing pattern size. (C) 2006 American Institute of Physics.
\end{abstract}

[DOI: $10.1063 / 1.2206247]$

While the nanolithographies based on tips as in atomic force microscopy, on beams as in electron-beam lithography, and on photons as in photolithography can be used for patterning, unconventional lithographies ${ }^{1-3}$ based on a mold have emerged as fast and simple methods that are applicable to large area. In the sub-100 $\mathrm{nm}$ range that is of interest in nanolithography, imprint lithography has been shown effective among the unconventional lithographies although it requires a high pressure and an extremely flat substrate surface. ${ }^{4}$ Composite poly(dimethylsiloxane) (PDMS) molds have been used to generate a pattern with a step height of $2 \mathrm{~nm}$ for $250 \mathrm{~nm}$ space $^{5}$ and a flat step structure with step height of $0.4 \mathrm{~nm} .{ }^{6}$ Only recently has capillary force lithography (CFL) been ushered into the sub-100 nm range with the introduction of a permeable Teflon mold. ${ }^{7}$

There has always been a prerequisite to the mold that is used for CFL, which is the permeability of the mold with respect to gas. If the mold is impermeable, the air trapped in the voids between the mold and the substrate surface gets compressed as the voids get filled by capillary action, which prevents the capillary rise. A basic question raised is whether the mold can be an impermeable hard mold and yet the CFL or the capillary rise can be effective. When a hard mold such as silicon mold is used for CFL, no capillary rise takes place for relatively large feature sizes as one might expect. In fact, it has been shown experimentally ${ }^{8}$ that no capillary rise occurs for a feature size of several hundred microns when the hard mold is used.

In this letter, we present capillary force lithography (CFL) in which impermeable hard mold is used. The use of hard mold such as silicon wafer implies that the feature size can be reduced to any that can be made on the hard mold. Furthermore, the smaller the feature size is, the more favorable the condition for patterning becomes for CFL.

The driving force for the capillary rise in a capillary of radius $r$ is the Laplace pressure $P_{L}$ that is given by

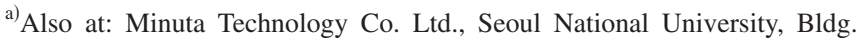
105-1, Room 212.

${ }^{b)}$ Also at: School of Mechanical and Aerospace Engineering and Institute of Advanced Machinery and Design, Seoul National University.

${ }^{c)}$ Author to whom correspondence should be addressed; electronic mail: honghlee@snu.ac.kr
}

$$
P_{L}=\frac{2 \gamma \cos \theta}{r}
$$

where $\gamma$ is the surface tension and $\theta$ is the contact angle that the material filling up the pore makes with the capillary. An implicit understanding here is that the contact angle is acute. The relationship also holds for line and space patterns, provided the radius is replaced with the channel width $L$ of the pattern. If the capillary rise takes place at atmospheric pressure and the capillary rise is to a height of $z$ in a capillary tube of length $Z$ (see Fig. 1), the pressure of the trapped air in the tube $P_{a}$ is given by $P_{0} Z /(Z-z)$ or $P_{0} /\left(1-z_{m}\right)$, where $z_{m}$ is $z / Z$ and $P_{0}$ is the initial air pressure ( $1 \mathrm{bar}$ ). The capillary rise will cease when $P_{a}$ equals the Laplace pressure. Therefore, the normalized height $z_{m}$ to which the material of interest rises at atmospheric pressure (1 bar) is given by

$$
z_{m}=1-\frac{1}{P_{L}}
$$

where $P_{L}$ is in bar unit. The geometric factor $r / 2$ in Eq. (1) is the ratio of the exposed surface area to the circumference that is in contact with the wall. When a channel infinitely long is involved, the geometric factor becomes $L / 2$, where $L$ is the channel width. Therefore, Eq. (1) applies to the channel if $r$ is replaced by $L$. As the relationship shows, there is no capillary rise taking place if the Laplace pressure is less than 1 bar. On the other hand, Eq. (1) tells us that the Laplace pressure increases inversely with the capillary radius or equivalently with the feature size of a pattern and that as

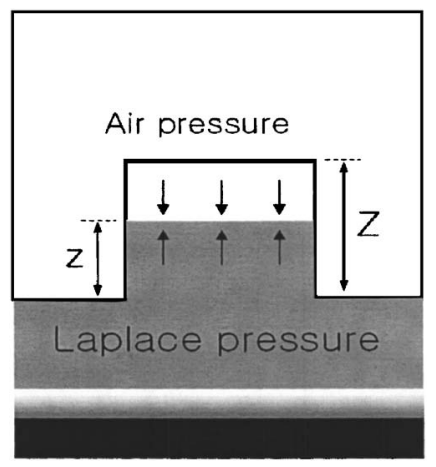

FIG. 1. Schematic illustration of capillarity and pertinent coordinate. 
the radius decreases, the pressure should exceed 1 bar. Therefore, CFL should be possible with impermeable hard mold.

If CFL were possible as the simple theory suggests, there would not have been nanoimprint lithography ${ }^{1}$ (NIL) in its present form since no pressure needs to be applied. The experience with NIL amply demonstrates that the capillary force is inoperative in that a high pressure close to 100 bars is needed for the patterning, the mechanism of which is squeezed flow ${ }^{9}$ of polymer melt. A natural question that arises is why CFL is ineffective with a hard mold on a hard substrate whereas the simple capillary theory says it could. The answer lies in the intimate contact between the mold and the substrate that is required of any capillary rise. Without the intimate contact, the material of interest simply cannot wet the capillary wall and hence no capillary rise can take place. When a hard mold is pressed against a hard substrate as in NIL, the intimate contact cannot be made all over the area being pressed because of the inherent roughness present on the mold as well as on the substrate. The pressure needed for the intimate contact all over the pressed area, which is also necessary for NIL, is so high that it far exceeds the Laplace pressure and the squeezed flow ensues. Therefore, the capillary force is inconsequential. In step and flash imprint lithography (SFIL), ${ }^{10}$ which is a form of NIL, a droplet of ultraviolet curable liquid is dispersed onto a base polymer layer on a hard substrate and then it is squeezed with a hard mold. Since the applied pressure is low, the capillarity must have played a role.

The intimate contact problem that is at the root can be resolved if a flexible mold is used. An example is poly(dimethylsiloxane) (PDMS). Because of the flexibility, it makes conformal contact with the underlying layer even if the surface is uneven and rough. However, the mold is not rigid enough that the mold pattern deforms laterally and the roof collapses when the feature size is small. ${ }^{11}$ Recently introduced is poly(urethaneacrylate) (PUA) mold ${ }^{12}$ that is rigid enough for fine patterning and yet flexible when it is made in a film form. In fact, this impermeable PUA mold has been shown to be rigid enough to be used for NIL. ${ }^{13}$ If a rigid and yet flexible mold allows intimate contact such that CFL can be carried out, conversely a completely hard mold such as the one made with silicon wafer can also be used for CFL, provided the substrate is flexible.

Shown in Fig. 2(a) is the master from which PUA mold has been made in a film form. ${ }^{12}$ The master pattern is an array of tapered cylinders with flat top. The bottom diameter is $200 \mathrm{~nm}$, the top diameter is $50 \mathrm{~nm}$, and the height is $800 \mathrm{~nm}$. The patterning result by CFL is shown in Fig. 2(b). For the patterning, polystyrene (PS) was spin coated onto the silicon wafer that had been coated with an adhesive layer and then dried. The adhesive layer was used so as to prevent the polymer layer from being detached from the substrate upon removing the mold. The adhesion layer was novolac resin in propylene glycol methyl ether acetate (PGMEA) that was mixed with an adhesion promoter of silane-based material. Onto this layer, polystyrene in toluene was spin coated. The PUA mold was then placed on the coated substrate, followed by a buffer PDMS block and then a steel plate. The weight of the plate is such that the applied pressure is approximately $100 \mathrm{~g} / \mathrm{cm}^{2}$ or about $0.1 \mathrm{bar}$, which was sufficient for intimate contact. The temperature was then raised to $150{ }^{\circ} \mathrm{C}$ using a hot stage. After cooling to room temperature, the Downloaded 26 Jun 2006 to 147.46.130.202. Redistribution subject
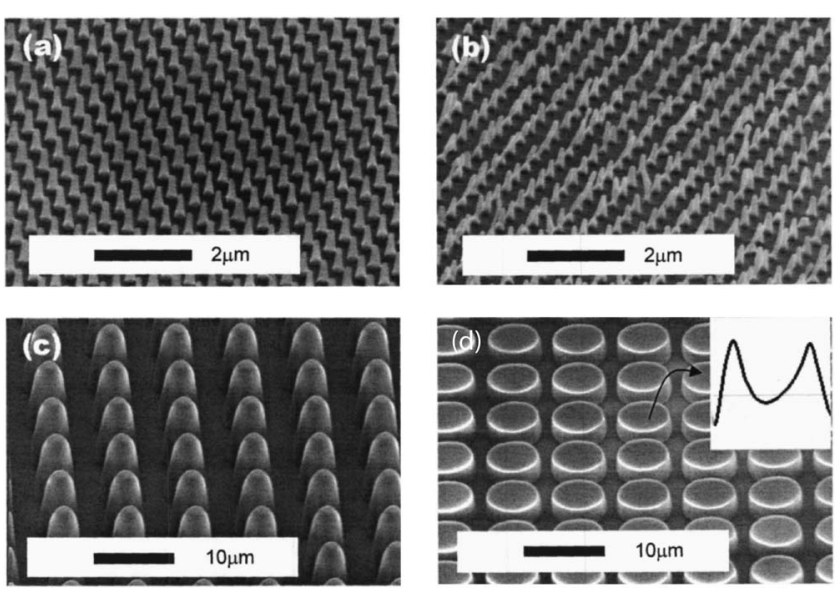

FIG. 2. SEM images of the masters and patterning results. (a) The master pattern is an array of tapered cylinders (the bottom diameter is $200 \mathrm{~nm}$ and the top diameter is $50 \mathrm{~nm}$ ). (b) The patterning result by CFL with impermeable PUA mold from the master in (a). (c) The master pattern is an array of slightly tapered cylinders (the bottom diameter is $6 \mu \mathrm{m}$ and the height is $10 \mu \mathrm{m})$. (d) The patterning result by CFL with impermeable PUA mold from the master in (c). It has a diameter of $5.78 \mu \mathrm{m}$ and a depth at the center of $865 \mathrm{~nm}$. The inset shows the sectional AFM image.

PUA mold was removed to obtain the result in Fig. 2(b). No antiadhesion layer was used for easy detachment.

To determine the contact angle and thereby ascertain the level of capillary pressure as a function of feature size, a master shown in Fig. 2(c) was used, the pattern of which is an array of slightly tapered cylinders. For the purpose, the pattern of large feature size was used such that the bottom diameter is $6 \mu \mathrm{m}$ and the height is $10 \mu \mathrm{m}$. The patterning result obtained with PS and the PUA mold made from the master are shown in Fig. 2(d). The CFL was carried out at $150{ }^{\circ} \mathrm{C}$ for $18 \mathrm{~h}$. As can be seen shortly, little capillary rise should have occurred because the diameter is not small enough for sufficient capillary pressure. However, some of the trapped air usually leaks out over a long period of time $(18 \mathrm{~h})$ and thus some capillary rise did occur and the height in Fig. 2(d) is $1.5 \mu \mathrm{m}$ as opposed to the master pattern height of $10 \mu \mathrm{m}$.

According to the sectional atomic force microscopy (AFM) result, the meniscus formed in Fig. 2(d) has a diameter of $5.78 \mu \mathrm{m}$ and a depth at the center of $865 \mathrm{~nm}$. The contact angle thus determined from the relationship $h=(1$ $-\sin \theta) D / 2 \cos \theta$ is $56.6^{\circ}$, which is the contact angle of PS with the PUA mold. Here $h$ is the meniscus depth at the center and $D$ is the diameter. Based on this contact angle, the capillary pressure can be calculated from Eq. (1) with $\gamma_{\mathrm{PS}-\text { air }}=40 \mathrm{mN} / \mathrm{m}$ as a function of the pattern size, as shown in Fig. 3. The result in Fig. 3 shows that the capillary pressure for the system under consideration exceeds the atmospheric pressure (1 bar line in Fig. 3 ) for the feature size smaller than about $440 \mathrm{~nm}$. Therefore, the capillary rise should ensue at atmospheric pressure for the feature size smaller than $440 \mathrm{~nm}$. While this result for the critical size $L_{C}$ was obtained for a particular set of materials, it can be determined in general from the relationship $L_{C}=2 \gamma \cos \theta$.

Another patterning result by CFL with the PUA mold is shown in Figs. 4(a)-4(c). The CFL was carried out at $150{ }^{\circ} \mathrm{C}$ with PS. The master shown in Fig. 4(a) is an $80 \mathrm{~nm}$ line/ $60 \mathrm{~nm}$ space pattern made of aluminum. The patterning result imaged by scanning electron microscopy (SEM) is to AlP license or copyright, see http://apl.aip.org/apl/copyright.jsp 


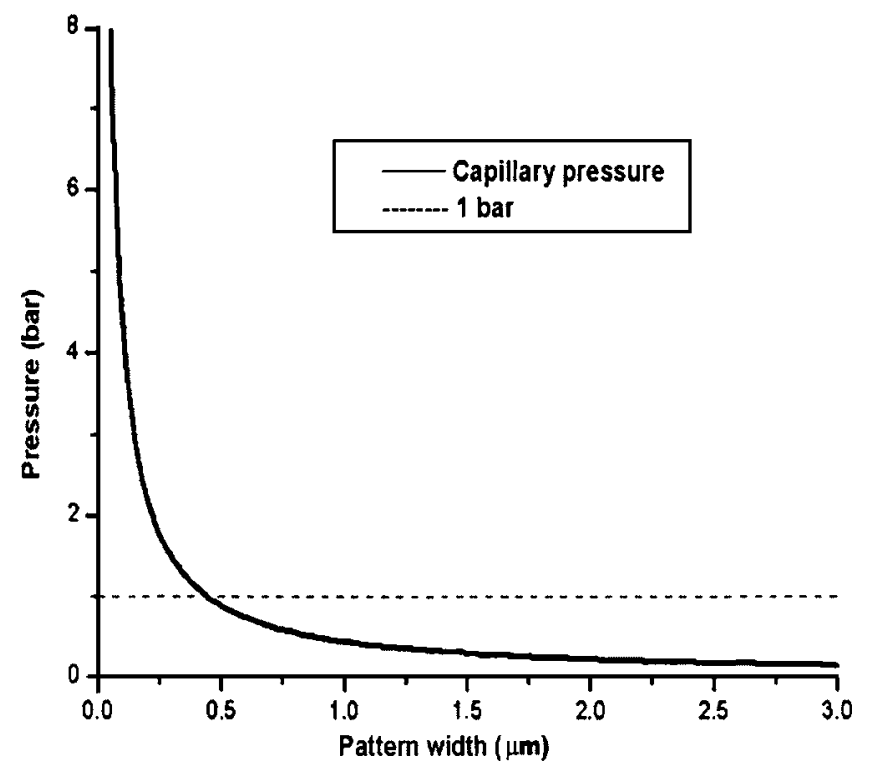

FIG. 3. Capillary pressure as a function of pattern size. For the feature size smaller than about $440 \mathrm{~nm}$, the capillary pressure exceeds the atmospheric pressure (1 bar) and the capillary rise should ensue.

shown in Fig. 4(b) and the cross sectional SEM micrograph of Fig. 4(b) in Fig. 4(c). It can be seen from Fig. 4(c) that the PS pattern formed has a height of approximately $150 \mathrm{~nm}$. For the $80 \mathrm{~nm}$ wide channel in the PUA mold, the corresponding capillary pressure is about 5.5 bars. According to Eq. (2), capillary rise should be $139 \mathrm{~nm}$ since the channel depth is $170 \mathrm{~nm}$. This calculated capillary rise compares with
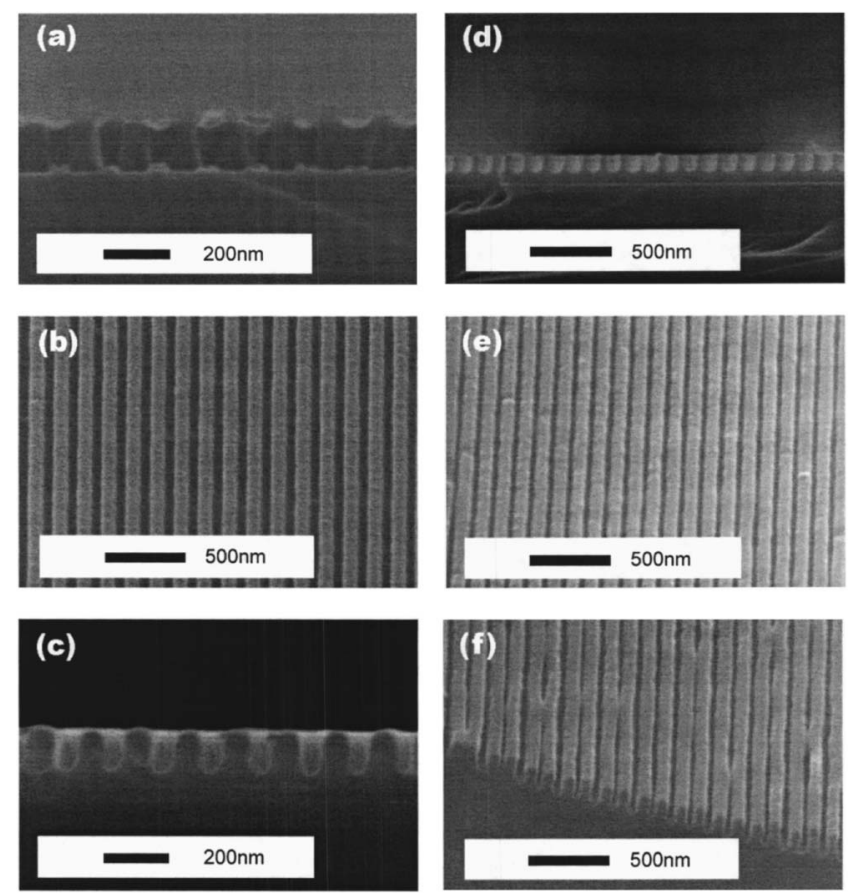

FIG. 4. SEM images of masters and patterning results. (a) The cross sectional image of master pattern with an $80 \mathrm{~nm}$ line/60 $\mathrm{nm}$ space pattern made of aluminum. [(b) and (c)] The plan and cross sectional SEM images, respectively, of PS patterning results by CFL with impermeable PUA mold (height is approximately $150 \mathrm{~nm}$ ). (d) The cross sectional image of master pattern made in the nitride layer on a silicon wafer shows a $30 \mathrm{~nm}$ line and $80 \mathrm{~nm}$ space pattern with a pattern depth of $120 \mathrm{~nm}$. [(e) and (f)] The plan and tilted SEM images, respectively, of the patterning result by CFL with PS and the silicon master in (d) as the mold. the experimental result of about $150 \mathrm{~nm}$ in Fig. 4(c).

As indicated at the outset, an impermeable and inflexible hard mold such as silicon mold can also be used for CFL provided the substrate is flexible. The mold used for the purpose is silicon wafer with a silicon nitride layer. The master pattern made in the nitride layer is a $30 \mathrm{~nm}$ line and $80 \mathrm{~nm}$ space pattern with a pattern depth of $120 \mathrm{~nm}$, as shown in Fig. 4(d). The substrate was flexible polyethylene terephthalate (PET) that was coated with PS. Onto this substrate, the silicon wafer mold was placed and a pressure of 0.1 bar was applied to ensure intimate contact between the mold and the substrate. The CFL was carried out at $150{ }^{\circ} \mathrm{C}$ for $10 \mathrm{~min}$. Note that the pattern formed in the PS layer is the negative of the mold pattern since the mold is the master itself such that the line is $80 \mathrm{~nm}$ wide and the space between lines is $30 \mathrm{~nm}$. The SEM image of the patterning result is shown in Fig. 4(e) and its tilted image in Fig. 4(f). The CFL carried out for $5 \mathrm{~min}$ led to essentially the same result, indicating that the actual time for the capillary rise would be shorter than the typical 10 min duration that was used in all the experiments.

In summary, capillary force lithography with impermeable mold has been presented. It has been found that nanosize patterning over a large area is possible with impermeable mold, contrasting the known fact that the micron-size patterning is impossible with impermeable mold when capillary force is mainly responsible for the patterning. The critical size below which such patterning is possible can be determined a priori. This nanosize patterning is possible only when either the impermeable mold or the substrate is flexible. When the substrate is flexible, which is relevant to the patterning for flexible devices and displays, rigid molds such as silicon wafer can be used. Therefore, the smallest feature size that can be patterned in this case could reach sub-10 nm range although the result demonstrated here is only for a dense pattern with a space of $30 \mathrm{~nm}$ between lines. The strength of CFL with impermeable mold lies in the fact that the natural driving force for the patterning, which is capillary pressure, increases with decreasing feature size.

This work was supported by the Korea Foundation Grant funded by MOEHRD (KRF-2005-041-D00250).

${ }^{1}$ S. Y. Chou, P. R. Krauss, and P. T. Reustrom, Science 272, 85 (1996).

${ }^{2}$ Y. Xia and G. M. Whitesides, Angew. Chem., Int. Ed. 37, 550 (1998).

${ }^{3}$ K. Y. Suh, Y. S. Kim, and H. H. Lee, Adv. Mater. (Weinheim, Ger.) 13, 1386 (2001).

${ }^{4}$ S. Y. Chou, P. R. Krauss, W. Zhang, L. J. Guo, and L. Zhuang, J. Vac. Sci. Technol. B 15, 2897 (1997).

${ }^{5}$ B. D. Gates and G. M. Whitesides, J. Am. Chem. Soc. 125, 14986 (2003).

${ }^{6}$ Q. Xu, B. T. Meyers, M. Lahau, D. V. Vezenov, and G. M. Whitesides, J.

Am. Chem. Soc. 1227, 854 (2005).

${ }^{7}$ D. Y. Khang and H. H. Lee, Langmuir 20, 2445 (2004).

${ }^{8}$ D. Y. Khang and H. H. Lee, Adv. Mater. (Weinheim, Ger.) 16, 176 (2004).

${ }^{9}$ H. C. Scheer, H. Scheelz, T. Hoffmann, and C. M. Sotomayer Torres, Handbook of Thin Film Materials (Academic, San Diego, 2001), Vol. 5, pp. 1-60.

${ }^{10}$ T. Baily, B. Smith, B. J. Choi, M. Colbum, M. Meisel, S. V. Sreenivasar, J. G. Eckerdt, and C. G. Wilson, J. Vac. Sci. Technol. B 19, 2806 (2001).

${ }^{11}$ E. Delamarche, H. Schmid, B. Michel, and H. Biebuyck, Adv. Mater. (Weinheim, Ger.) 9, 741 (1997); H. Schmidt and B. Michel, Macromolecules 33, 3042 (2000).

${ }^{12}$ S. Choi, P. J. Yoo, S. J. Baek, T. W. Kim, and H. H. Lee, J. Am. Chem. Soc. 126, 7744 (2004).

${ }^{13}$ P. J. Yoo, S. Choi, J. H. Kim, D. Suh, S. J. Baek, T. W. Kim, and H. H. Lee, Chem. Mater. 16, 5000 (2004). 